\title{
Epicardial fat volume is associated with coronary endothelium-dependent vasomotor response in healthy subjects
}

\author{
Bénédicte Gaborit ${ }^{1 *}$, Pierre Julien Moro ${ }^{2}$, Antonin Flavian ${ }^{3}$, Frank Kober ${ }^{3}$, Alexis Jacquier $^{3}$, Jacques Quilici ${ }^{4}$, \\ Thomas Cuisset ${ }^{5}$, Umberto Simeoni ${ }^{6}$, Patrick Cozzone ${ }^{3}$, Monique Bernard ${ }^{3}$, Anne Dutour ${ }^{1}$
}

From 2011 SCMR/Euro CMR Joint Scientific Sessions

Nice, France. 3-6 February 2011

\section{Introduction}

Epicardial fat $\left(E_{\text {fat }}\right)$ is an active ectopic fat depot, directly surrounding coronary arteries, and secreting high level of inflammatory adipokines; its development has been associated with coronary atherosclerosis. We investigated the relationship between $E_{\text {fat }}$ and endothelium dependent vasoreactivity of the coronary microcirculation.

\section{Methods}

Myocardial blood flow (MBF) was determined by measuring coronary sinus flow with velocity-encoded cine magnetic resonance imaging at 3 teslas. We measured MBF at baseline and in response to sympathetic stimulation by cold pressor testing (CPT) in 17 healthy volunteers with normal left ventricular function (age 24 \pm 6 years, $\mathrm{BMI}=21.1 \pm 2.6 \mathrm{~kg} / \mathrm{m} 2)$. $\mathrm{E}_{\mathrm{fat}}$ volume was volumetrically assessed by manual delineation on short-axis views. CPT was applied by immersing one foot in ice water for 4 minutes.

\section{Results}

A significant increase in MBF was observed: $1.18 \pm 0.58$ vs $0.84 \pm 0.47 \mathrm{~mL} . \mathrm{min}-1 . \mathrm{g}-1, \mathrm{CPT}$ vs rest, $\mathrm{p}=0.002$. Mean relative MBF increase $(\triangle \mathrm{MBF})$ was $50 \pm 47 \%$. Mean $\mathrm{E}_{\mathrm{fat}}$ volume was $82 \pm 31 \mathrm{~mL}$ and varied from 43 to $131 \mathrm{~mL}$; mean LV mass and Left ventricular ejection fraction were $104 \pm 31 \mathrm{~g}$ and $64 \pm 5 \%$, respectively. CPT significantly increased heart rate (HR) by $28 \pm 13 \%$, systolic blood pressure (BP) by $17 \pm 13 \%$, diastolic BP by $23 \pm 19 \%$ and rate-pressure

${ }^{1}$ INSERM U626, Marseille, F-13385 France, Centre de Résonance Magnétique Biologique et Médicale (CRMBM), CNRS UMR 6612, Department of Endocrinology, Metabolic Diseases and Nutrition, CHU Nord, Marseille,

France, Marseille, France

Full list of author information is available at the end of the article product by $52 \pm 25 \%, \mathrm{p}<0.01$, indicating an increase in myocardial work load. The increase in HR, reflecting sympathetic stimulation, was not influenced by sex, age or $\mathrm{E}_{\mathrm{fat}}$ volume. CPT induced a decrease in coronary vascular resistance $(150 \pm 93$ vs $114 \pm 44 \mathrm{mmHg} . \mathrm{mL}-1 . \mathrm{min} . \mathrm{g})$ by trend $(\mathrm{p}=0.08)$. Interestingly, we found a significant negative correlation between $\mathrm{E}_{\text {fat }}$ volume and $\triangle \mathrm{MBF}(\mathrm{r}=-0.51$, $\mathrm{p}=0.03$ ), which remained significant after adjusting for age and sex. $\triangle \mathrm{MBF}$ was not associated with waist circumference, BMI, CRP, lipid or glycemic parameters.

\section{Conclusion}

The increase in $E_{\text {fat }}$ is associated with a decrease in endothelium dependent vasoreactivity response, suggesting that $E_{\text {fat }}$ could early influence endothelial function.

\section{Author details}

${ }^{1}$ INSERM U626, Marseille, F-13385 France, Centre de Résonance Magnétique Biologique et Médicale (CRMBM), CNRS UMR 6612, Department of Endocrinology, Metabolic Diseases and Nutrition, CHU Nord, Marseille, France, Marseille, France. ${ }^{2}$ Centre de Résonance Magnétique Biologique et Médicale, CNRS UMR N6612, Marseille, France. ${ }^{3}$ Centre de Résonance Magnétique Biologique et Médicale (CRMBM), CNRS UMR 6612 "Marseille, France. ${ }^{4}$ Department of Cardiology, CHU Timone, Marseille, France, Marseille, France. ${ }^{5}$ INSERM U626, Marseille, F-13385 France, Department of Cardiology, CHU Timone, Marseille, France, Marseille, France. ${ }^{6}$ Department of Neonatalogy, Children and Parents Pole, CHU Timone, Marseille, France, Marseille, France.

Published: 2 February 2011

doi:10.1186/1532-429X-13-S1-P71

Cite this article as: Gaborit et al: Epicardial fat volume is associated with coronary endothelium-dependent vasomotor response in healthy subjects. Journal of Cardiovascular Magnetic Resonance 2011 13(Suppl 1): P71. 